\title{
Design Optimization of Amazon Robotics
}

\author{
Jun-tao Li ${ }^{1}$, Hong-jian Liu ${ }^{2}$ \\ ${ }^{1}$ School of Information, Beijing Wuzi University, Beijing, China \\ ${ }^{2}$ Graduate Department, Beijing Wuzi University, Beijing, China
}

Email address:

ijuntao@bwu.edu.cn (Jun-tao Li), 522927485@qq.com (Hong-jian Liu)

\section{To cite this article:}

Jun-tao Li, Hong-jian Liu. Design Optimization of Amazon Robotics. Automation, Control and Intelligent Systems. Vol. 4, No. 2, 2016, pp. 48-52. doi: 10.11648/j.acis.20160402.17

Received: May 4, 2016; Accepted: May 14, 2016; Published: May 30, 2016

\begin{abstract}
In the era of e-commerce, the logistic distribution center is put in the center role of order picking for the sake of meeting the needs of different customer orders, hence, improving the automation and work capacity of distribution center becomes research priority in the fields of logistics and warehousing. The objective of this article is to-solve the shortcomings of currently traditional distribution center picking system with high automation by introducing a new method of picking provided by Amazon's Kiva system, that is, mobile racking with goods is broke out to sorting table by Kiva mobile robots named AGVs (automated guided vehicles), which could not only reduce the walk time and labor cost, but improve efficiency. This article starts with the constitutive requirements of the Amazon KIVA robotic systems, and then some key problems of Kiva picking system and design optimization about task allocation and path planning of multi-robots are demonstrated respectively in this article. Finally, the content is summarized and the application of robotic system in is simply demonstrated and prospected.
\end{abstract}

Keywords: Amazon Kiva Robotics, Picking System, Design Optimization

\section{Introduction}

In the operation procedure of distribution center, picking is considered as the primary field to improve operation efficiency of warehousing as it occupies plentiful funds which accounts for fifty percent of overall distribution center operating costs. As one of the largest multinational E-company, Amazon has created a new logistics model that could be called "shelf to the people" by using Kiva robotic system during the US holiday season last year. In Massachusetts, a city of America, Quiet Logistics is located as a big Operations Center with area of 25,500 square meters [1]. In this distribution center, there are 200 robots to help workers deal with 10thousands to 20 thousand order items per day. With the help of these robot, the overall efficiency of picking system would improve by 3.5 times to 5 times. In this new picking system, robots search for the mobile racking full of products based on the bar code and then move to the front of the review packager directly, then items required on this order were chosen by a review packager to make secondary sorting and packaging review, etc. Amazon employees just standing in a fixed point to complete the whole work, that is to say, the timing cost of pickers spent walking could be reduced close to zero in Kiva system. Kiva robotic system could not only reducing unnecessary labor costs, but its thin fuselage and highly efficient route planning also increase the storage space by reducing the passage area to improve throughput of the overall system. Thus, the emergence of Kiva robots better meets the needs of E-company to efficiently handle a large number of diversified orders effectively and relieve the order delivery pressure of $s$ that the electricity commercial companies faced with during the holiday season such as "Black Friday" [2].

Compared with the traditional distribution center, Amazon's Kiva system design a new type of multi-robot system for order picking instead of ATS (automatization tridimensional storehouse), long distance belt conveyor, Carousel, etc. Kiva robot is only $76 \mathrm{~cm}$ long, $64 \mathrm{~cm}$ wide and $41 \mathrm{~cm}$ high. Because of its own limitations, the mobile rack is designed very small (5 layers) and each layer could only put 2-3 items. Kiva robot could carry a ton of items in spite of its small size. When order signal is received, the robot was sent to ship the mobile rack with required items to the picking zone [3]. These robots could even find their charging stations to replenish their energy when they are about to run out of energy. These robots could work in an orderly manner 
without collision, mainly due to the manipulation of the software [2]. Therefore, time cost of the robotic system could significantly be reduced and picking efficiency could be improved by optimizing the allocation of freight tasks and their work and return path through the information system.

At present, There is more attention on the design optimazation of traditional distribution center picking system with high automation [4], but a little research about kiva robot at home and abroad, and kiva robot algorithms has not been public, therefore, this article will focus on the design optimization about task allocation and path planning of multi-robots, which represents some theoretical value.

\section{Design of Amazon Robotics}

Amazon Robotics is designed for the sake of meeting growing consumer demands in e-commerce by using a better system to provide order fulfillment solutions. As the wholly owned subsidiary of Amazon.com, Amazon Robotics makes it more convenience, more effective, faster in e-Commerce order real-time processing through automation. Most advanced robotic technology is used to achieve highly automated distribution center operations. These methods includes autonomous mobile robots, sophisticated control software, language perception, power management, computer vision, depth sensing, machine learning, object recognition, and semantic understanding of commands.

In particular, a complete robotic picking system (Goods to person or Goods to man, G2P or G2M) consists of three parts [5]:

\subsection{The Back End Workstation System}

This system uses distributed software / hardware architecture, firstly, information about items and suppliers is stored in the back end system in each warehouse. This kind of warehouse storage is generally in a random fashion, and SKU is placed in a appropriate position in storage according to the frequency of shipments of each SKU, in the meantime, high-speed real-time storage is realized; secondly, order processing is accomplished by connecting with ERP (Enterprise Resource Planning) and WMS (warehouse management system).

The best route of picking is automatically planned when the order information arrives to autonomous mobile robots and then the most appropriate picking station is automatically searched, in the same time, the order processing sequence is also calculated automatically; afterwards, adequately scheduling and dispatching robot resource becomes a notable feature to make a plurality of robots running at the same time work in an orderly space and try to avoid the queues, cross-collision and other conditions, while the charging time is scheduled according to assignments of each robot.

\subsection{The High-Speed Mobile Communication System}

The high-speed mobile communication system is used to guarantee stable wireless network in the warehouse and also to avoid the "offline hour" during the picking work.

\subsection{Autonomous Mobile Robots}

It is generally believed that the height form factor is an important factor to consider in the design of robots. Robots is considered that it would run more stable if it was designed shortly, as a result, Kiva robots are $40 \mathrm{~cm}$ high. Kiva's autonomous mobile robots apparatus comprising:

Table 1. Design of Amazon AGV.

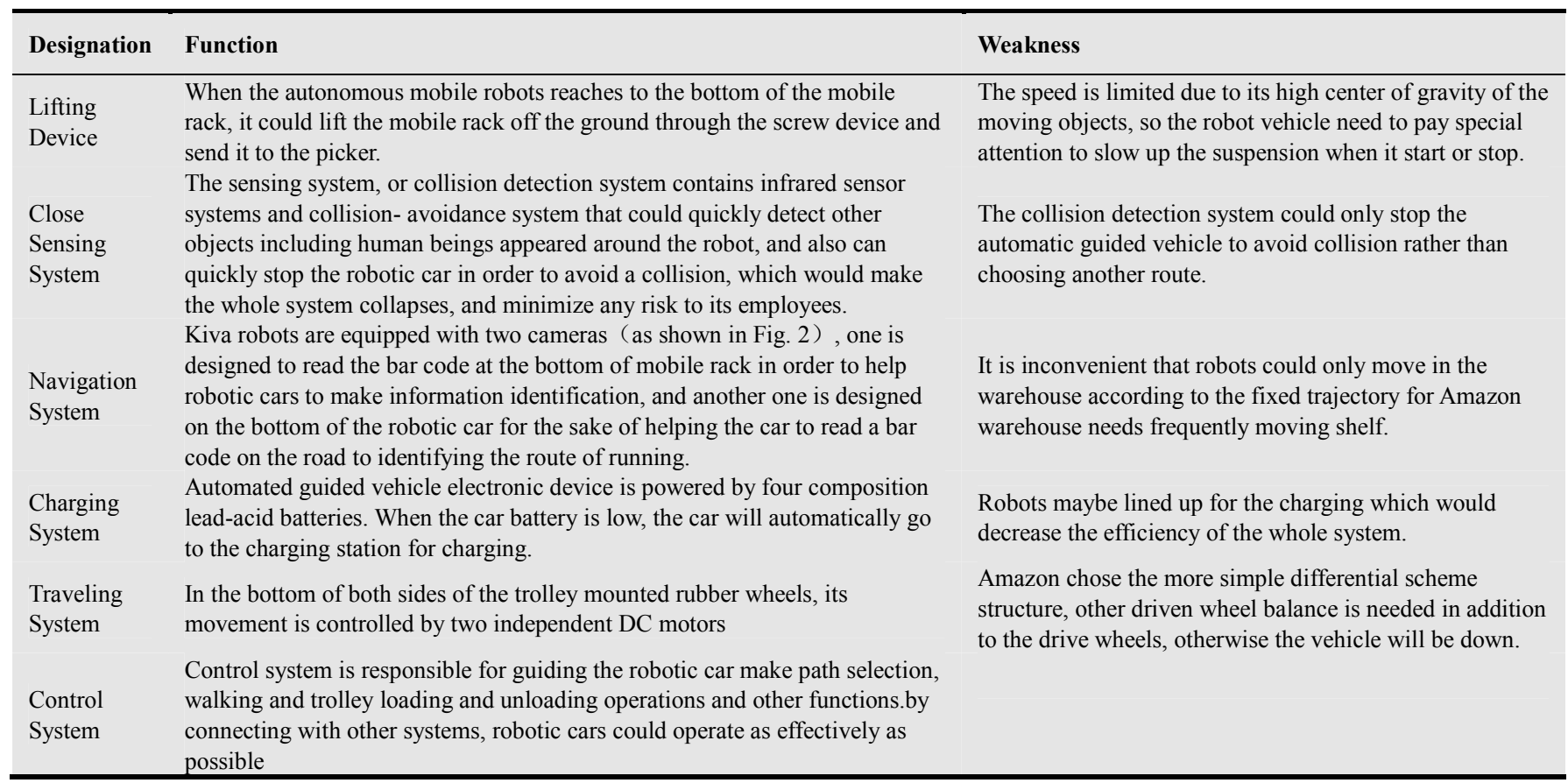




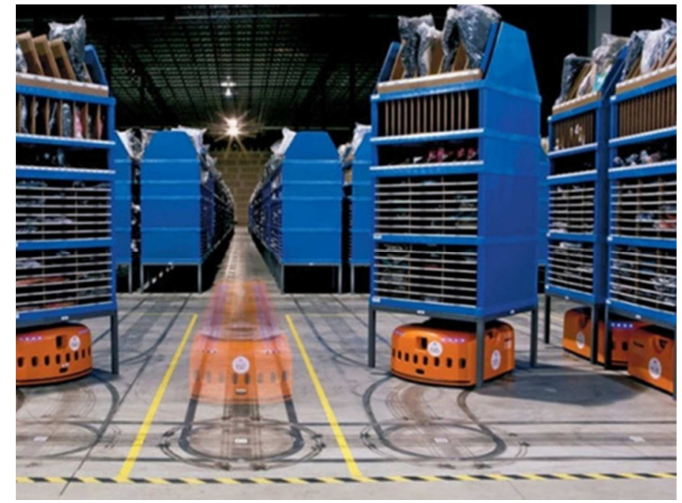

Figure 1. Navigation System of Amazon Robotics.

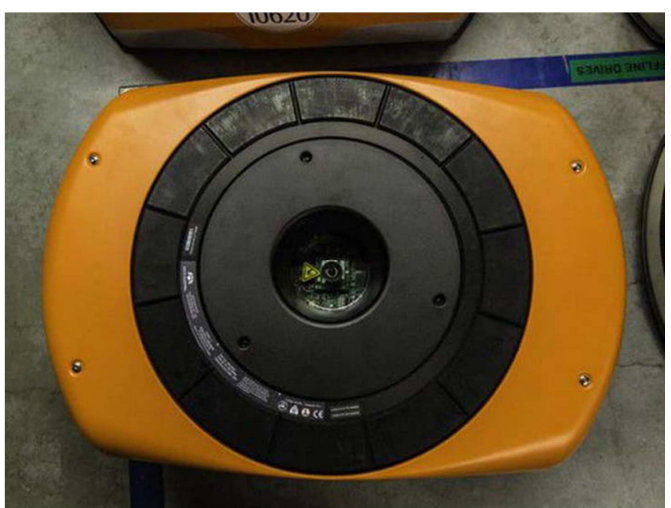

Figure 2. Kiva robots are equipped with cameras.

\section{Key Issues in Kiva Robot Operation}

The most advanced robot design of Amazon's Kiva picking system is that it can run accurately by following the identification code on the ground in distribution center, so as to avoid mistakes caused by collision between each other. At the same time, robotic cars are designed that there would be no more than 10 a robot fails at the same time in each layer of the warehouse; therefore, the working accuracy of the Amazon robots can reach to $99.99 \%$ [6], which could greatly reduce the probability of human error. When kiva robot receiving orders of picking, they will line up under setting rules and came to picking area and then pickers would remove the goods. Finally, Kiva robots follow the path back to the storage area to wait for the next picking work.

Kiva system perfect make the connection between the new and advanced artificial intelligence technology and logistics automation technology, and it also make the integrated use of computer systems, robotic car, the shuttle shelves and workstations and other basic tools to achieve automation of picking process in distribution center. And specifically, the Kiva robot operation mode is summarized as follows:

(1) Receive and process orders

These Kiva robots are both fast and quiet in positioning the aimed mobile rack where contains the items in the picking order after it receiving the picking order information, and the picking orders are assigned to different order picking stations. After these robots received the wireless transmission of digital commands from central computer, by scanning a bar code label on the ground through the sliding shelf to the ground, then $1.2 \mathrm{~m}$ wide, $340 \mathrm{~kg}$ heavy shelve is lifted [7]. Amazon's picking robots are equipped with the advanced visual system that can use bar codes to track every product on the shelf, at the time fetch the appropriate shelves when order pickers coming to.

(2) Complete tasks and get back

When the picking is finished, the information of finishing tasks would be sent to the system and then the system determines the new storage location of mobile rack based on the current overall situation and sent the results to the robots to select return routine, and then the robotic car will return back corresponding to the storage location. This position is selected is the result of re-calculation under normal circumstances as a reason why it is different from the previous shelf position.

(3) Be released or charged.

The robot will be released and continue to accept new tasks after it placed mobile rack back to the storage area [8]. Meanwhile, during the system operation time, the robot remaining charge will also be real-time monitored and low battery robotic car will be guided to a charging station. When charging is completed, robots continue to return to the system operation.

\section{Design Optimization of Kiva Robot}

Kiva robot complete the order task in four steps which is shown in Figure 2.

Step 1. Kiva robots receive orders, and go to the position of the mobile rack under selection.

Step 2. Kiva robots transport the mobile rack from the original location to the site into a queue and to prepare to be selected.

Step 3. Orders are satisfied by picked, packaged and re-checked by Pickers

Step 4. The mobile rack which has been picked would be sent back to a now storage location by Kiva robots.

As can be seen from figure 3, design optimization of Amazon's kiva robot could become a reality in the following three aspects:

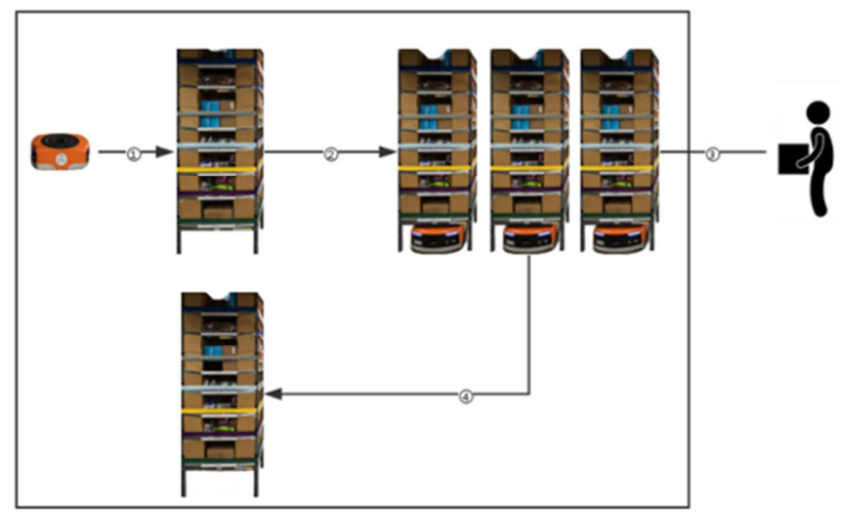

Figure 3. Four steps of Kiva robot complete the order task. 


\subsection{Task Allocation of Multi-Robots}

In distribution center applied Kiva system, automatic mobile robots should be designed aimed to avoid robot in spare time to ensure the robot to perform tasks in the full time [9]. These robots in a time window could have three different kind of states: (1) performing tasks; (2) be released into the next round when task is finished; (3) Waiting for performing task. In this optimization, the robots only have the first two states in a perfect working environment that are performing tasks and be released into the next round. Therefore, the optimization of multi-robots task allocation can effectively reduce time cost in step one, and then improve the working efficiency of the entire system.

In Kiva Systems, mobile racks are arranged in the area of storage in a regular way to prepare to be available for robots to find by its definite position, coordinates, and bar code [10]. Task allocation of multi-robots is optimized by comprehensively considering arrival sequence and position of robots under the mobile rack as well as the status of each robot, and robotic cars match the nearly mobile rack in task in the system:

Assume that within a window of time for the process of order picking, during this time, the task store shelves in order of arrival of the waiting shelf, waiting for the robotic car to come to work. Mobile rack that to be processed are considered as set S1 $\{\mathrm{M} 1, \mathrm{M} 2, \mathrm{M} 3, \mathrm{M} 4, \mathrm{M} 5 \ldots\}$, while robot at rest in the current time as set $\mathrm{S} 2\{\mathrm{R} 1, \mathrm{R} 2, \mathrm{R} 3, \mathrm{R} 4, \mathrm{R} 5 \ldots\}$. The current positions of each Mobile rack that to be processed and robotic car at rest in S1 and S2 are known. Consider arrival sequence and position of robots under the mobile rack as well as the status of each robot to make the task allocation. the specific method to Determine the task allocation is to consider the Manhattan Distance between not allocated mobile rack with all current free AGVs, and the nearest AGV whose Manhattan Distance is smallest would be chosen to suit for the not allocated mobile rack and then update the set S1 and set S2 in this system, and then the next task sequentially selecting a free trolley shelf in accordance with the method described above.

For example, assuming orders in a time window has been processed, the task mobile rack to be picking are lined up in the order, that is M1, M2, M3, M4, M5..., From the first one to the last, M1 come to the first to be selected the robotic car in free. Assuming that there are 5 automated robotic vehicle, $\mathrm{R} 1, \mathrm{R} 2, \mathrm{R} 3, \mathrm{R} 4, \mathrm{R} 5$, the nearest car is chosen to perform this task shelves by comparison task Manhattan distance between $\mathrm{M} 1$ and robotic vehicles. If the R2 is calculated to be the nearest one in Manhattan Distance, it would be matched with the task of M1, that is to say, task allocation is completed between $\mathrm{M} 1$ and $\mathrm{R} 2$. When there are more than one robotic cars have the smallest Manhattan Distance with M1, there will be a short time judgment of the car number and the smallest number would be selected by the controlling system to perform the current task. The number of elements in set S2 will be updated and then mobile rack continue to be allocated. All the mobile racks are assigned task after end of work.
This type of task allocation model gives full consideration about the sequence of mobile rack in task, that is priority of shelf and it also consider the current form for all robotic vehicle in the system. The task allocation is finished based on the nearest Manhattan Distance between robotic cars and mobile racks which make the picking system performs tasks with a shorter time and smaller walking path.

\subsection{Path Planning of Multi-Robots}

After completing picking task, the robot needs to carry the mobile rack to shelf storage area. In the process of return, new storage location is produced with following different path of return. By considering and rank the frequency the mobile racks are chosen, the higher frequency mobile rack would be placed in a closer position to the platform while the low frequency mobile rack would be placed in a further location that away from the site, and picking tasks are finished through the information systems by real-time monitoring and real-time adjustments.

The design optimization of the Path planning of multi-robots would not only to further enhance the Kiva robot's walking efficiency in the operation process, but also to provide a lot of room for improvement for the path optimization in the next process [11].

\section{Summary and Prospect}

In the robotics ecosystem, Amazon Robotic comes up with the new automated picking solutions. In this picking system Kiva robots can automatically arrive to mobile rack and lifted it to the picking station according to the order needs and inventory information. Pickers are prompted for which items in the order should be picked from the mobile rack; all of his work is reaching for items without walking, which greatly enhance the level of automation of distribution centers. At present, Google, Apple and other technology giants have set foot in the field of robotics [12]. Currently, China's e-commercial enterprises have also made efforts in robotic innovation to follow and bring cutting edge technology into the logistic field. For instance, About a dozen of Geek + robots are put into use in Tmall (Tianjin) warehouse smart picking area of 2000 square meters in year 2015 during the holiday season. It is believed that there are greater possibilities of optimization and a better application foreground.

\section{Acknowledgements}

This study is supported by intelligent logistics system Beijing Key Laboratory (NO: BZ0211) and Beijing Intelligent Logistics System Collaborative Innovation Center.

\section{References}

[1] News \& Trends. "Amazon is equipped with robots to meet holiday season". World vision, vol. 23, pp. 36-37, 2014. 
[2] Industry dynamics. "Amazon use robots handle orders". Journal of Robot Technique and Application, vol 1, pp58-60, 2015.

[3] X. M. Zhang. "The order picking optimization and algorithm research based on Kiva system," Master Thesis, Beijing: Beijing University of Posts and Telecommunications, 2015.

[4] D'Andrea R, Wuman P. Future challenges of coordinating hundreds of autonomous vehicles in distribution facilities//Techonologies for Practical Robot Applications, 2008. TePRA2008. IEEE International Conference on. IEEE, pp 80-83, 2008.

[5] J. P. Wu. "Analysis and prospect of Amazon kiva robot application," Journal of Logistics \& Material Handling, vol. 10, pp 159-162, 2015.

[6] M. Zamirian, A. V. Kamyad, M. H. Farahi. "A novel algorithm for solving optimal path planning problems based on parametrization method and fuzzy aggregation," Journal of Physics Letters A. 2009 (38).
[7] P. Klaus. Logistics research: a 50 years' march of ideas. Logistics Research. 2009.

[8] J. Enright, P. R. Wurman. "Optimization and Coordinated Autonomy in Mobile Fulfillment Systems. Automated Action Planning for Autonomous Mobile Robots,” 2011.

[9] S. Qi. "Study on path planning of AGV system," Master Thesis, Zhejiang: Zhejiang University, 2012.

[10] R. Peter. Wurman, Raffaello D'Andrea, and Mick Mountz. "Coordinating hundreds of cooperative, autonomous vehicles in warehouses," Journal of AI Magazine, vol 29, no. 1, 2008.

[11] Ruey-Jer "Bryan" Jean, R. R Sinkovics, D. Kim. "The impact of technological, organizational and environmental characteristivs on electronic collaboration and relationship performance in international customer-supplier relationships," Journal of Information and Management, 2014.

[12] T. Frank, C. Q. Zhang. "To the robot era of apple, amazon and Google," Journal of World Science, vol 3, pp 53-54, 2014. 